\title{
Medications and Supplements Prescription Patterns During COVID 19 Pandemic in Yemen: A Questionnaire-Based Study
}

Abdullah Chahin ( $\sim$ abdullah_chahin@brown.edu )

Warren Alpert Medical School of Brown University: Brown University Warren Alpert Medical School https://orcid.org/0000-0001-8209-4984

Ghulam Dhabaan

University of Toronto

Abdulrahman Buhaish

N/A

Mahmoud Shorman

The University of Tennessee Health Science Center Department of Medicine

\section{Research Article}

Keywords: COVID-19, Yemen, Coronavirus, Covid-19, pandemic, treatment, supplement, prescription patterns.

Posted Date: April 7th, 2021

DOl: https://doi.org/10.21203/rs.3.rs-369104/v1

License: (1) This work is licensed under a Creative Commons Attribution 4.0 International License. Read Full License 


\section{Abstract}

Introduction: the study aims to better understand the COVID-19 prescription treatments and over the counter regimens in Yemen in view of limited published data and limited availability of COVID-19 testing.

Methods: A 34 question web-based survey was distributed on social media outlets targeting people in Yemen. Data aggregation, analysis, and visualization were performed using Tableau and Microsoft Excel.

Results: 2341 individuals reported symptoms concerning for COVID-19 infection, with 25.4\% reporting a chronic medical condition. Female patients were less likely to receive medications for treatment in all age groups examined. Azithromycin was the most prescription medication prescribed (32.8\%) and vitamin C being the most supplement used (62\%). Around 5.5\% were on Hydroxychloroquine prophylaxis prior to their diagnosis and only $12.9 \%$ of them continued using after diagnosis.

Conclusions: This study provides some important information about the commonly observed treatments and prescription patterns during the COVID-19 pandemic in Yemen during May- July of 2020. The study reflects the influence of global trends in medication prescription even in resource-limited countries.

\section{Introduction}

Coronavirus disease (COVID-19) since it was first reported in December 2019, continues to present a global challenge especially with the rapidly increasing numbers of new cases worldwide, and the lack of definitive treatment [1],[2]. Globally, as of 18 January 2021, 93,805,612 confirmed cases of COVID-19 have been reported to the World Health Organization (WHO) including 2,026,093 deaths [3].

Due to the lack of cure, the management of COVID-19 infected patients continues to focus on supportive care provisions, including, maintaining oxygenation, ventilation, and proper fluid management [4]. The Infectious Diseases Society of America Guidelines on the Treatment and Management of Patients with COVID-19 recommends different options depending on clinical presentation and site of treatment including antiviral therapy, immunotherapy, and cellular therapy. However, these practice guidelines need to be updated frequently as most of these treatment options continue to be under investigation resulting in rapidly emerging published literature [5]. There are different treatment guidelines for COVID-19 worldwide depending on resources [1]. In this paper we report the results of an electronic questionnaire that was developed and distributed online among Yemeni people. We aim to better understand the COVID19 treatment and over the counter regimens in Yemen in view of limited published data and limited availability of COVID-19 testing.

\section{Methods}

A web-based questionnaire - with 34 questions and 56 data points - was designed using google forms. The questionnaire form was distributed to the public using social media outlets. No selection was made to the participants, aside from being In Yemen or from Yemen. Response from 4059 participants. The 
prescription medications were captured as check boxes for common regimens that the respondents can use. In addition, the respondents can enter the medications as free text. The data was cleaned, and some collected variables were transformed using OpenRefine and python. Data aggregation, analysis, and visualization were performed using Tableau and Microsoft Excel.

\section{Results}

The questionnaire captured 2341 individuals reported symptoms concerning for COVID-19 infection between May and July of 2020. In Table 1, we list some of the major characteristics of the population we surveyed. The most prevalent age group was $21-30$ years old and $51.7 \%$ were male. A sum of 595 individuals reported having a chronic medical illness (25.4\%). Obesity was the most reported chronic medical condition (233 respondents, 10\%), followed by hypertension (7.7\%) and diabetes (6.5\%). Asthma was reported as a chronic illness by $54(2.3 \%)$ respondents. As for substance use, we observed that $21.9 \%$ of the respondents reported tobacco usage, compared to $57.5 \%$ Gat usage.

Table 1

General Demographic Characteristics of the respondents.

\begin{tabular}{|lll|}
\hline Demographic Characteristic & $n$ & $\%$ \\
\hline Male gender & 1,210 & $51.7 \%$ \\
\hline Age group (Mode) & $21-30$ & $31.9 \%$ \\
\hline Chronic diseases & 595 & $25.4 \%$ \\
\hline Diabetes Mellitus & 151 & $6.5 \%$ \\
\hline Hypertension & 180 & $7.7 \%$ \\
\hline Obesity & 233 & $10.0 \%$ \\
\hline Asthma & 54 & $2.3 \%$ \\
\hline Hospitalized & 147 & $6.3 \%$ \\
\hline Developed respiratory failure requiring supplemental O2 or mechanical ventilation & 92 & $3.9 \%$ \\
\hline
\end{tabular}

We began our analysis by looking at the medication prescription patterns by age group and gender. Table 2 demonstrates the percentage of male patients receiving treatment was higher than females. It also shows the progressive tendency to receive treatment with increasing age (from $74.4 \%$ in children 10 years of age and younger to $94.7 \%$ for elderly age 71 and higher). The prescription percentage is certainly high, but the list of medications received includes many of the over-the-counter medications such as paracetamol (acetaminophen), vitamins, and zinc. When we look at the discrepancy between medication prescription in age, graph 1 shows that this discrepancy is seen among all age groups and it is most pronounced in early adulthood and middle age groups. The difference hovered around 7-10 percentage 
points. The widest gap of prescription intake between males and females was seen in the 61-70 Years age group ( $94.7 \%$ for males vs $84.4 \%$ for females).

Table 2

Prescription pattern by age group and gender

\begin{tabular}{|llll|}
\hline & Medication count (\%) & No meds count (\%) & Total \\
\hline Total & $2087(89.1 \%)$ & $254(10.9 \%)$ & 2341 \\
\hline Female & $977(86.4 \%)$ & $154(13.6 \%)$ & 1131 \\
\hline Male & $1110(91.7 \%)$ & $100(8.3 \%)$ & 1210 \\
\hline 1 Day - 10 Years & $71(74.7 \%)$ & $24(25.3 \%)$ & 95 \\
\hline $\mathbf{1 1 - 2 0}$ Years & $199(74.8 \%)$ & $67(25.2 \%)$ & 266 \\
\hline 21-30 Years & $684(91.7 \%)$ & $62(8.3 \%)$ & 746 \\
\hline 31-40 Years & $590(91.9 \%)$ & $52(8.1 \%)$ & 642 \\
\hline 41-50 Years & $284(92.8 \%)$ & $22(7.2 \%)$ & 306 \\
\hline $\mathbf{5 1 - 6 0}$ Years & $149(90.3 \%)$ & $16(9.7 \%)$ & 165 \\
\hline 61-70 Years & $74(89.2 \%)$ & $9(10.8 \%)$ & 83 \\
\hline 71 Years or older & $36(94.7 \%)$ & $2(5.3 \%)$ & 38 \\
\hline
\end{tabular}

Graph 1: Percentage of male patients receiving treatment vs female across age groups. (see Graph 1 in the Supplementary Files)

There were more than 30 different substances consumed therapeutically in our questionnaire (Table 3). They ranged from antibiotics and systemic steroids to vitamins and herbal remedies. We chose to focus on 7 treatment agents, which have had some data on their use as part of treatment for COVID-19. Those were: Hydroxychloroquine, Azithromycin, Vitamin C, Vitamin D, Zinc, Paracetamol, and systemic Corticosteroids. 
Table 3

List of medications, supplements, herbs and food that was reported as part of a regimen to treat COVID-19 infections

\begin{tabular}{|lll|}
\hline Midications & Supplements & Herbs and food \\
\hline Aspirin & Multivitamins & Honey \\
Guaifenesin & Vitamin B complex & Black seed \\
Solpadine (Paracetamol + Codeine) & Vitamin B12 & Lemon \\
Cefaclor & Selenium & Lime \\
Amoxicillin & Omega 3 & Clove \\
Ceftriaxone & & Costus \\
Levofloxacin & & Ginger \\
Ciprofloxacin & & Roselle \\
Antibiotic capsules & & Onions \\
Fraxiparine & & garlic \\
Enoxaparin & & Fruits \\
esomeprazole & & Vegetables \\
\hline
\end{tabular}

We wanted to look at prescription patterns among folks who were not hospitalized, in comparison to those who were admitted to the hospital and those who developed respiratory failure requiring Oxygen supplementation or mechanical intubation. Table 4 shows what percentage of patients received each of these treatments in the 3 clinical categories. Vitamin $\mathrm{C}$ was the most prescribed treatment in our database and ranged between $62 \%$ for non-hospitalized patients to $74 \%$ for hospitalized patients. Azithromycin was the second most commonly used agent, with a significant increase of percentage receiving the treatment with increasing severity (32.1\% for non-hospitalized, $57.1 \%$ for hospitalized, and $67.5 \%$ for patients with respiratory failure). Surprisingly, systemic corticosteroids and paracetamol were the least prescribed treatments, with only 192 individuals (8.2\%) receiving paracetamol and only 19 individuals $(0.8 \%)$ receiving systemic corticosteroids.

As for antibiotics other than hydroxychloroquine and azithromycin, the rate of prescription was surprisingly low. Only 31 participants (1.4\%) who were not hospitalized received an antibiotic, 3 of the hospitalized patients, and 2 of the patients who developed respiratory failure (2.1\%).

Except for paracetamol, all treatments were more commonly prescribed for hospitalized patients and patients with respiratory failure, compared to those who were not hospitalized. The likelihood of receiving those medications seemed to increase with increasing severity in general. 
Table 4

Frequency of medication prescribing by hospitalization status and respiratory failure.

\begin{tabular}{|lllll|}
\hline Medication & All & Outpatient (\%) & Hospitalized (\%) & Respiratory failure (\%) \\
\hline Hydroxychloroquine & $140(5.9 \%)$ & $117(5.3 \%)$ & $23(15.6 \%)$ & $19(24.7 \%)$ \\
\hline Azithromycin & $788(33.7 \%)$ & $704(32.1 \%)$ & $84(57.1 \%)$ & $52(67.5 \%)$ \\
\hline Vit C & $1451(62 \%)$ & $1,361(62.0 \%)$ & $90(61.2 \%)$ & $57(74.0 \%)$ \\
\hline Vit D & $767(32.8 \%)$ & $712(32.5 \%)$ & $55(37.4 \%)$ & $38(49.4 \%)$ \\
\hline Zinc & $504(21.5 \%)$ & $464(21.1 \%)$ & $40(27.2 \%)$ & $35(45.5 \%)$ \\
\hline Paracetamol & $189(8.0 \%)$ & $187(8.5 \%)$ & $2(1.4 \%)$ & $3(3.9 \%)$ \\
\hline Corticosteroids & $19(0.8 \%)$ & $13(0.5 \%)$ & $2(1.4 \%)$ & $4(5.2 \%)$ \\
\hline Other antibiotics & $34(1.5 \%)$ & $31(1.4 \%)$ & $3(2.0 \%)$ & $2(2.1 \%)$ \\
\hline
\end{tabular}

During the time this survey was conducted, the use of Hydroxychloroquine was a common practice for treating COVID-19 infection as well as for prophylaxis around the world. At the same time, more data was coming out about the lack of efficacy. We looked at the frequency of Hydroxychloroquine use among our sample and how many of them continued using it after diagnosis. We also were able to report on how many were started on Hydroxychloroquine after diagnosis. Table 5 shows that 128 individuals (5.5\%) were on Hydroxychloroquine prophylaxis prior to their diagnosis and only 18 of those patients $(12.9 \%)$ continued using the agent. On the other hand, we found that 122 patients (5.2\% of the study participants) were started on Hydroxychloroquine treatment after diagnosis. In total, 140 individuals reported receiving Hydroxychloroquine treatment for their COVID-19 infection (6\% of the participants).

Table 5

Hydroxychloroquine prescribing before and after the diagnosis.

\begin{tabular}{|c|c|c|c|c|}
\hline & & \multicolumn{3}{|c|}{$\begin{array}{l}\text { Was the patient on } \\
\text { Hydroxychloroquine? }\end{array}$} \\
\hline & & Yes $(\% \rightarrow)$ & No $(\% \rightarrow)$ & Total \\
\hline & & $(\% \downarrow)$ & $(\% \downarrow)$ & \\
\hline \multirow{5}{*}{$\begin{array}{l}\text { Was the patient prescribed Hydroxychloroquine after } \\
\text { diagnosis }\end{array}$} & Yes & 18 & \multirow{2}{*}{$\begin{array}{l}122(87.1 \%) \\
(5.5 \%)\end{array}$} & \multirow[t]{2}{*}{140} \\
\hline & & $(12.9 \%)$ & & \\
\hline & \multirow[t]{2}{*}{ No } & $\begin{array}{l}110 \\
(5.0 \%)\end{array}$ & $\begin{array}{l}2091 \\
(95.0 \%)\end{array}$ & \multirow[t]{2}{*}{2201} \\
\hline & & (85.9\%) & $(94.9 \% \rightarrow)$ & \\
\hline & Total & 128 & 2213 & 2341 \\
\hline
\end{tabular}


Hydroxychloroquine. Finally, we looked at the most common combinations of treatment agents in our database. Table 5 summarizes our findings. We found that $20.7 \%$ of participants took medications other than the 7 treatments we looked at. Vitamin $\mathrm{C}$ was the most common single agent used in the whole database, with $16.2 \%$ of the participants receiving it therapeutically. $12.2 \%$ reported receiving no treatment. The most common combination was azithromycin with vitamin C, with $10.2 \%$ of participants receiving it. We noted $7.9 \%$ of participants receiving vitamins $C$ and $D$ and $7.8 \%$ reporting they took azithromycin, vitamins $C$ \& D, and Zinc. Vitamin $C$ was used in the vast majority of the combinations (8 out of 12), with azithromycin and vitamin $D$ in second place (5/12 for each). Vitamins $C$ and $D$ were the most occurring pain in the combinations observed. The observed combinations accounted for more than $99 \%$ of the combinations observed in this study.

Table 6: Most common medication combinations prescribed.

\begin{tabular}{|c|c|c|c|c|c|c|c|c|}
\hline FALSE & FALSE & FALSE & FALSE & FALSE & FALSE & FALSE & 431 & $20.7 \%$ \\
\hline FALSE & FALSE & FALSE & TRUE & FALSE & FALSE & FALSE & 338 & $16.2 \%$ \\
\hline TRUE & FALSE & FALSE & FALSE & FALSE & FALSE & FALSE & 254 & $12.2 \%$ \\
\hline FALSE & FALSE & TRUE & TRUE & FALSE & FALSE & FALSE & 213 & $10.2 \%$ \\
\hline FALSE & FALSE & FALSE & TRUE & TRUE & FALSE & FALSE & 165 & $7.9 \%$ \\
\hline FALSE & FALSE & TRUE & TRUE & TRUE & TRUE & FALSE & 163 & $7.8 \%$ \\
\hline FALSE & FALSE & FALSE & TRUE & TRUE & TRUE & FALSE & 146 & $7.0 \%$ \\
\hline FALSE & FALSE & TRUE & TRUE & TRUE & FALSE & FALSE & 112 & $5.4 \%$ \\
\hline FALSE & FALSE & TRUE & FALSE & FALSE & FALSE & FALSE & 95 & $4.6 \%$ \\
\hline FALSE & FALSE & FALSE & FALSE & FALSE & FALSE & TRUE & 64 & $3.1 \%$ \\
\hline FALSE & TRUE & TRUE & TRUE & TRUE & TRUE & FALSE & 54 & $2.6 \%$ \\
\hline FALSE & FALSE & FALSE & TRUE & FALSE & FALSE & TRUE & 48 & $2.3 \%$ \\
\hline
\end{tabular}

\section{Discussion}

The data about COVID-19 pandemic in Yemen is limited, and studies are challenged by the lack of diagnostic testing, where in a prior study, only $1.6 \%$ of populations with clinical diagnosis of COVID-19 infection had their diagnosis confirmed with a PCR test [6].

COVID-19 prescription patterns and rate of medication and/or supplement intake has not been well described in medical literature. Although this is a questionnaire based observational study, our findings provide some interesting insights about prescription patterns, by demographics (sex and age groups) and by disease severity (outpatient, hospitalized, and with respiratory failure). It also looks at the trends of 
medications and supplements prescribing, and the most common combinations of medications used in a resource limited country.

Our study is unique in trying to shed light on the treatment disparities among sexes and age groups, especially in a war-torn country in the middle east in the setting of a pandemic. In the case of COVID-19 infection, it was clear early on the increased age was a risk factor and predictor of poor outcome in COVID-19 infection [7-9]. That is possibly the reason why we have noted a steady increase of the likelihood of receiving treatment with older age groups. As for the disparities in treatment among sexes, the data has been mixed. A meta-analysis from 2016 showed that Women were $27 \%$ more likely than men to receive an antibiotic prescription[10]. Pain management, on the other hand, seemed to have significant gender bias against women [11], despite the clear data that shows women pain is physiologically more pronounced than men [12].

This study was conducted in May of 2020, when there were several attempts to find effective treatment for the COVID-19 infection. At that point, the data on using agents such as hydroxychloroquine, azithromycin, vitamin $D$, and others to help treat the infection and decrease the severity of the illness has failed to demonstrate a statistically significant change. The literature on prescription and usage patterns for agents other than antibiotics in COVID-19 infection remains scarce. Our study also looked at systemic corticosteroids treatment months prior to the RECOVERY trial was published, at a time when corticosteroids usage was still controversial [13].

In accordance with the global trend, the use of hydroxychloroquine and azithromycin was remarkable in our study population. Eventually, both medications were not shown to be effective in COVID-19 treatment [14]. Around one third of the respondents who were treated as outpatients received antibiotics, a rate similar to previously published studies [15]. Our study also highlighted the increased tendency to prescribe antimicrobial to hospitalized patients, where two thirds of the patients received antibiotics, which has been similar to what has been reported in literature $(72 \%-90 \%[16,17])$.

Out of the vitamins and minerals we looked at in our study, vitamin C was the most commonly used supplement in this study, with $62 \%$ receiving it alone or as part of their treatment regimen. This might be due to the historical trend of consuming vitamin $\mathrm{C}$ in cases of influenza and upper respiratory tract infection. Vitamin D came second, with $32.8 \%$ of respondents receiving it as therapy. This study predates the body of literature that signaled a worse outcome with vitamin D deficiency and COVID-19 infection [18]. Zinc came third, with $21.5 \%$ of the respondents being treated with. Indirect observational evidence suggests zinc may potentially reduce the risk, duration and severity of COVID-19 infections, especially in cases of zinc deficiency [19].

Finally, the rate of using two anti-inflammatory agents' treatments: paracetamol (acetaminophen) and systemic steroids was explored in this study. The utilization of paracetamol was surprisingly low ( $8 \%$ total, with decreased frequency of usage with increased clinical severity). This is probably due to the initial warnings of the potential harmful effects of using anti-inflammatory medications in COVID-19 infections at the beginning of the pandemic [20]. That previous insight probably contributed to the low 
utilization of systemic steroids as well ( $1.5 \%, 2 \%$ for admitted patients), especially before the results of the RECOVERY trial were published in July of 2020.

This study has several limitations: one of these is being an online questionnaire-based study. It also did not include diagnostic tests such as real-time polymerase chain reaction or lung computed tomography results.

\section{Conclusion}

There seems to be a strong influence of emerging publications on the prescription of antibiotics and antiinflammatory medications in the case of suspected COVID-19 infection in Yemen. The frequent use of supplementary vitamins and minerals as part of treatment for COVID-19 has not been reported on before and this study has quantified the abundance of their use along with the most commonly prescribed combinations.

Transparency declaration

The lead author affirms that this manuscript is an honest, accurate, and transparent account of the study being reported; that no important aspects of the study have been omitted; and that any discrepancies from the study as planned (and, if relevant, registered) have been explained.

\section{Declarations}

Transparency declaration

The lead author affirms that this manuscript is an honest, accurate, and transparent account of the study being reported; that no important aspects of the study have been omitted; and that any discrepancies from the study as planned (and, if relevant, registered) have been explained.

Authors Contributions

All authors contributed equally to this work.

Funding

This study did not receive any funding.

Ethical Approval and Consent to participate

the need for approval was waived.

Consent for publication 
I, the undersigned, give my consent for the publication of identifiable details, which can include graph(s), table(s), and/or details within the text ("Material") to be published in the above Journal and Article.

Availability of data and materials

The dataset is available upon request.

Competing interests

The authors have no conflict of interests to declare.

Acknowledgements

All contributors to the project were recognized as authors.

Authors' information

Abdullah Chahin, MD, MA, MSc

Department of Medicine

Warren Alpert School of Medicine at Brown University

Abdullah_Chahin@Brown.edu

Ghulam Dhaban, PhD

Department of Microbiology

University of Toronto

ghulam.dhabaan@utoronto.ca

Abdulrahman Buhaish

Independent researcher

ghulam.dhabaan@gmail.com

Mahmoud Shorman, MD, MHA

Department of Medicine

The University of Tennessee Health Science Center Department of Medicine mshorman@utmck.edu 


\section{References}

1. Lai CC, Shi TP, Ko WC, Tang HJ, Hsueh PR. Severe acute respiratory syndrome coronavirus 2 (SARSCoV-2) and coronavirus disease-2019 (COVID19): The epidemic and the challenges. Int J Antimicrob Agents. 2020:105924. https://doi.org/10.1016/j.jjantimicag.2020.105924.

2. Hin Fung Tsang, Lawrence Wing Chi Chan, William Chi Shing Cho, Allen Chi Shing Yu, Aldrin Kay Yuen Yim, Amanda Kit Ching Chan, Lawrence Po Wah Ng, Yin Kwan Evelyn Wong, Xiao Meng Pei, Marco Jing Woei Li \& Sze-Chuen Cesar Wong (2020) An update on COVID-19 pandemic: the epidemiology, pathogenesis, prevention and treatment strategies, Expert Review of Anti-infective Therapy, DOI: 10.1080/14787210.2021.1863146.

3. (Coronavirus Disease (COVID-19). Available online: https://www.who.int (accessed on 18 January 2021)

4. Cunningham, A.C., Goh, H.P. \& Koh, D. Treatment of COVID-19: old tricks for new challenges. Crit Care 24, 91 (2020). https://doi.org/10.1186/s13054-020-2818-6.

5. www.idsociety.org/COVID19guidelines. Accessed on 7 January, 2021

6. Dhabaan G, Chahin A, Buhaish A, Shorman M. COVID-19 pandemic in Yemen: A questionnaire based survey, what do we know? J Infect Dev Ctries. 2020 Dec 31;14(12):1374-1379. doi:

10.3855/jidc.13966. PMID: 33378278.

7. Chen T., Dai Z., Mo P., Li X., Ma Z., Song S., Chen X., Luo M., Liang K., Gao S., et al. Clinical characteristics and outcomes of older patients with coronavirus disease 2019 (COVID-19) in Wuhan, China (2019): A single-centered, retrospective study. J. Gerontol. Ser. A. 2020 doi: 10.1093/gerona/glaa089.

8. Dudley J.P., Lee N.T. Disparities in Age-specific Morbidity and Mortality From SARS-CoV-2 in China and the Republic of Korea. Clin. Infect. Dis. 2020;71:863-865. doi: 10.1093/cid/ciaa354.

9. Romero Starke K, Petereit-Haack G, Schubert M, et al. The Age-Related Risk of Severe Outcomes Due to COVID-19 Infection: A Rapid Review, Meta-Analysis, and Meta-Regression. Int J Environ Res Public Health. 2020;17(16):5974. Published 2020 Aug 17. doi:10.3390/ijerph17165974

10. Wiebke Schröder, Harriet Sommer, Beryl Primrose Gladstone, Federico Foschi, Jenny Hellman, Birgitta Evengard, Evelina Tacconelli, Gender differences in antibiotic prescribing in the community: a systematic review and meta-analysis, Journal of Antimicrobial Chemotherapy, Volume 71, Issue 7, July 2016, Pages 1800-1806.

11. Anke Samulowitz, Ida Gremyr, Erik Eriksson, Gunnel Hensing, "'Brave Men" and "Emotional Women": A Theory-Guided Literature Review on Gender Bias in Health Care and Gendered Norms towards Patients with Chronic Pain", Pain Research and Management, vol. 2018, Article ID 6358624, 14 pages, 2018. https://doi.org/10.1155/2018/6358624.

12. Berkley KJ. Sex differences in pain. Behav Brain Sci. 1997;20:371-380.

13. RECOVERY Collaborative Group, Horby P, Lim WS, Emberson JR, Mafham M, Bell JL, Linsell L, Staplin N, Brightling C, Ustianowski A, Elmahi E, Prudon B, Green C, Felton T, Chadwick D, Rege K, Fegan C, 
Chappell LC, Faust SN, Jaki T, Jeffery K, Montgomery A, Rowan K, Juszczak E, Baillie JK, Haynes R, Landray MJ. Dexamethasone in Hospitalized Patients with Covid-19 - Preliminary Report. N Engl J Med. 2020 Jul 17:NEJMoa2021436. doi: 10.1056/NEJMoa2021436. Epub ahead of print. PMID: 32678530; PMCID: PMC7383595.

14. Cavalcanti AB, Zampieri FG, Rosa RG, Azevedo LCP, Veiga VC, Avezum A, Damiani LP, Marcadenti A, Kawano-Dourado L, Lisboa T, Junqueira DLM, de Barros E Silva PGM, Tramujas L, Abreu-Silva EO, Laranjeira LN, Soares AT, Echenique LS, Pereira AJ, Freitas FGR, Gebara OCE, Dantas VCS, Furtado RHM, Milan EP, Golin NA, Cardoso FF, Maia IS, Hoffmann Filho CR, Kormann APM, Amazonas RB, Bocchi de Oliveira MF, Serpa-Neto A, Falavigna M, Lopes RD, Machado FR, Berwanger O; Coalition Covid-19 Brazil I Investigators. Hydroxychloroquine with or without Azithromycin in Mild-to-Moderate Covid-19. N Engl J Med. 2020 Nov 19;383(21):e119. doi: 10.1056/NEJMx200021.

15. Pulia MS, Wolf I, Schwei RJ, et al. Antibiotic prescribing patterns for coronavirus disease 2019 (COVID-19) in two emergency departments with rapid procalcitonin [published online ahead of print, 2020 Nov 19]. Infect Control Hosp Epidemiol. 2020;1-3. doi:10.1017/ice.2020.1329

16. Rawson TM, Moore LSP, Zhu N, et al. Bacterial and Fungal Coinfection in Individuals With Coronavirus: A Rapid Review To Support COVID-19 Antimicrobial Prescribing. Clin Infect Dis. 2020;71(9):2459-2468. doi:10.1093/cid/ciaa530

17. Youngs J, Wyncoll D, Hopkins $P$, Arnold A, Ball J, Bicanic T. Improving antibiotic stewardship in COVID-19: Bacterial co-infection is less common than with influenza. J Infect. 2020;81(3):e55-e57. doi:10.1016/j.jinf.2020.06.056.

18. Pereira M, Dantas Damascena A, Galvão Azevedo LM, de Almeida Oliveira T, da Mota Santana J. Vitamin D deficiency aggravates COVID-19: systematic review and meta-analysis. Crit Rev Food Sci Nutr. 2020 Nov 4:1-9. doi: 10.1080/10408398.2020.1841090. Epub ahead of print. PMID: 33146028.

19. Arentz S, Hunter J, Yang G, et al. Zinc for the prevention and treatment of SARS-CoV-2 and other acute viral respiratory infections: a rapid review. Adv Integr Med. 2020;7(4):252-260. doi:10.1016/j.aimed.2020.07.009

20. Day M. Covid-19: ibuprofen should not be used for managing symptoms, say doctors and scientists. BMJ. 2020 Mar 17;368:m1086. doi: 10.1136/bmj.m1086. PMID: 32184201.

\section{Supplementary Files}

This is a list of supplementary files associated with this preprint. Click to download.

- Graph1.pdf 